\title{
Reduction of Unphysical Wave Reflection Arising From Space-Time Finite Integration Method
}

\author{
$\operatorname{AUTHOR}(\mathrm{S})$ : \\ Matsuo, Tetsuji; Mifune, Takeshi
}

\section{CITATION:}

Matsuo, Tetsuji ...[et al]. Reduction of Unphysical Wave Reflection Arising From SpaceTime Finite Integration Method. IEEE Transactions on Magnetics 2014, 50 (2): 177-180

\section{ISSUE DATE: \\ 2014-02 \\ URL: \\ http://hdl.handle.net/2433/187795 \\ RIGHT:}

(C) 2014 IEEE. Personal use of this material is permitted. Permission from IEEE must be obtained for all other uses, in any current or future media, including reprinting/republishing this material for advertising or promotional purposes,

creating new collective works, for resale or redistribution to servers or lists, or reuse of any copyrighted component of this work in other works.; This is not the published version. Please cite only the published version.; この論文は出版社版

でありません。引用の際には出版社版をご確認ご利用ください。 


\title{
Reduction of Unphysical Wave Reflection Arising from Space-Time Finite Integration Method
}

\author{
Tetsuji Matsuo and Takeshi Mifune, Members, IEEE
}

Graduate School of Engineering, Kyoto University, Kyoto 615-8510 Japan

\begin{abstract}
Several 3D and 4D space-time grids are compared for electromagnetic wave computations using the space-time finite integration (FI) method. To suppress unphysical wave reflections without incurring numerical instability, the constitutive relation is corrected while retaining the symmetric of impedance matrix. The eigenvalue analysis confirms the stability of the 3D space-time FI schemes, where an asymmetric impedance matrix could cause late instability. A 4D space-time FI scheme is also improved by symmetric corrections.
\end{abstract}

Index Terms-Computational electromagnetics, finite difference methods, numerical stability, time domain analysis.

\section{INTRODUCTION}

$\mathrm{T}$ HE FINITE integration (FI) method [1]-[3] provides timedomain electromagnetic wave computation on an unstructured spatial grid. Refs. [4], [5] developed a space-time FI method that realizes non-uniform time-steps on 3D and 4D space-time grids to relax the Courant-Friedrichs-Lewy condition with respect to the smallest spatial grid size, which was applied to an optical device analysis [6]. Refs. [7] and [8] proposed improved 3D and 4D space-time grids to suppress unphysical wave-reflections caused by a nonuniform spatial grid construction [4], [5]. However, the stability of these schemes has not been confirmed for very long computations that might suffer late-time instability [9].

This paper proposes an improved constitutive relation based on a vector correction, where the stability of the resultant scheme is evaluated by an eigenvalue analysis. Computational accuracy resulting from several space-time grids proposed previously are compared.

\section{Finite Integration Method on A Space-Time Grid}

The coordinate system is denoted by $(c t, x, y, z)=\left(x^{0}, x^{1}, x^{2}\right.$, $\left.x^{3}\right)$ where $c=1 / \sqrt{ }\left(\varepsilon_{0} \mu_{0}\right)$, and $\varepsilon_{0}$ and $\mu_{0}$ are the permittivity and permeability of the vacuum. The integral forms of the Maxwell equations without source terms are [5]:

$$
\begin{aligned}
& \oint_{\partial \Omega \mathrm{p}} F=0, \oint_{\partial \Omega \mathrm{d}} G=0, \\
& F=-\sum_{i=1}^{3} E_{i} \mathrm{~d} x^{0} \mathrm{~d} x^{i}+\sum_{j=1}^{3} \mathscr{B}_{j} \mathrm{~d} x^{k} \mathrm{~d} x^{l}, \\
& G=\sum_{i=1}^{3} H_{i} \mathrm{~d} x^{0} \mathrm{~d} x^{i}+\sum_{j=1}^{3} \mathscr{D}_{j} \mathrm{~d} x^{k} \mathrm{~d} x^{l}
\end{aligned}
$$

where $\left(\mathscr{B}_{1}, \mathscr{B}_{2}, \mathscr{B}_{3}\right)=c \boldsymbol{B}$ and $\left(\mathscr{D}_{1}, \mathscr{D}_{2}, \mathscr{D}_{3}\right)=c \boldsymbol{D} ;(j, k, l)$ is a cyclic permutation of $(1,2,3) ; \Omega_{\mathrm{p}}$ and $\Omega_{\mathrm{d}}$ are hypersurfaces in space-time. The electromagnetic variables are defined in the FI method as:

$$
f=\int_{\mathrm{Sp}} F, \quad g=\int_{\mathrm{Sd}} G
$$

where $S_{p}$ and $S_{d}$ are the faces of the primal and dual grids that constitute $\partial \Omega_{\mathrm{p}}$ and $\partial \Omega_{\mathrm{d}}$. The Hodge dual grid [5] is used to express the constitutive equation simply as:

Manuscript received June 25, 2013. Corresponding author: T. Matsuo (email: tmatsuo@kuee.kyoto-u.ac.jp).

Digital Object Identifier inserted by IEEE

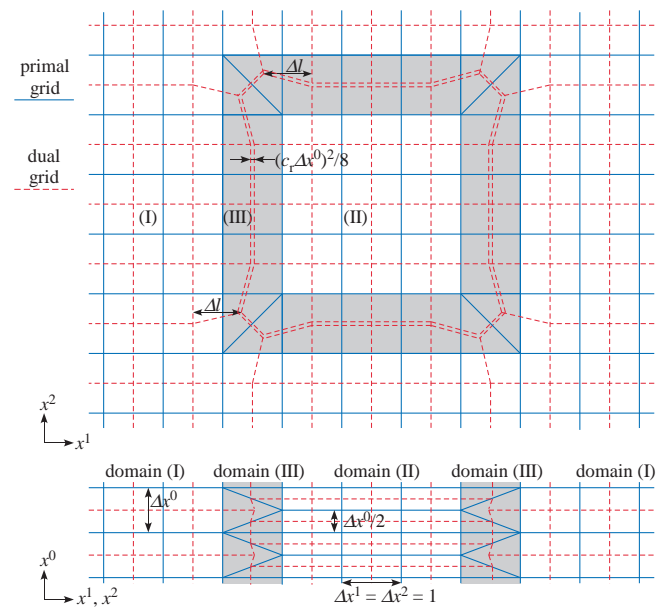

Fig. 1. Space-time grid (solid line: primal grid, dashed lines: dual grid)

$$
\frac{\int_{\mathrm{Sd}} c_{\mathrm{r}} \mathrm{d} x^{0} \mathrm{~d} x^{j}}{\int_{\mathrm{Sp}} \mathrm{d} x^{k} \mathrm{~d} x^{l}}=-\frac{\int_{\mathrm{Sd}} \mathrm{d} x^{k} \mathrm{~d} x^{l}}{\int_{\mathrm{Sp}} c_{\mathrm{r}} \mathrm{d} x^{0} \mathrm{~d} x^{j}}=a
$$

where $c_{\mathrm{r}}=1 / \sqrt{ }\left(\varepsilon_{\mathrm{r}} \mu_{\mathrm{r}}\right), a$ is a constant determined for each pair of $S_{\mathrm{p}}$ and $\mathrm{S}_{\mathrm{d}} ; \varepsilon_{\mathrm{r}}$ and $\mu_{\mathrm{r}}$ are the relative permittivity and permeability. Thus, $f=Z g / a$ is obtained, where $Z=$ $\sqrt{ }\left(\mu_{\mathrm{r}} \mu_{0} / \varepsilon_{\mathrm{r}} \varepsilon_{0}\right)$ is the impedance.

\section{3D-SPACE-TIME GRIDS WITH 2D SPACE}

\section{A. 3D-Space-Time Grids}

Fig. 1 illustrates the space-time grids proposed in this paper, where domains (I) and (II) have uniform time-steps $\Delta x^{0}$ and $\Delta x^{0} / 2$, respectively; domain (III) is the connecting domain. For simplicity, the spatial cell size is set to 1 by normalization. Fig. 2(a) and (b) shows the grid structure of corner parts of domain (III) examined in [7] and [8] to suppress the unphysical wave reflection due to spatial irregularity. In this article, the grids shown in Figs. 1, 2(a), and 2(b) are called types A, B, and C, respectively. Fig. 3 illustrates the three types of corner for domain (III).

Similar to a type-B grid, a type-A grid has dual edges that are not orthogonal to the corresponding primal faces (Fig. 1), where (4) is not exactly applicable. This study examines two types of constitutive relations described by (5) and (6): 


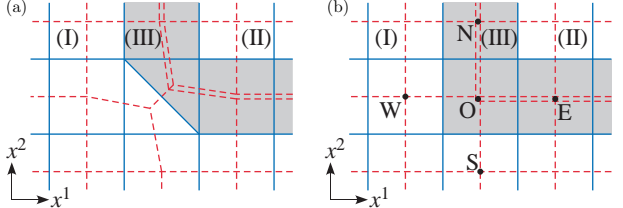

Fig. 2. Corner of domain (III): (a) type-B grid and (b) type-C grid.

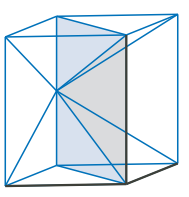

Type A Type B
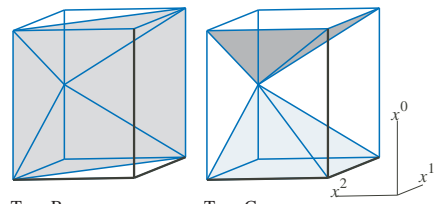

Fig. 3. Comparison of three types of corner for domain (III)

$$
\begin{aligned}
& e_{i \mathrm{I}}^{n+1 / 2}=Z c_{\mathrm{r}} \Delta x^{0} \frac{d_{i \mathrm{I}}^{n+1 / 2}}{\Delta l}, e_{i \mathrm{II}}^{n+1 / 2}=Z \frac{c_{\mathrm{r}} \Delta x^{0}}{2} \frac{d_{i \mathrm{I}}^{n+1 / 2}}{\Delta l} \\
& e_{i \mathrm{II}}^{n+j / 4}=Z \frac{c_{\mathrm{r}} \Delta x^{0}}{4} \frac{d_{i \mathrm{II}}^{n+j / 4}}{\Delta l}, \quad(i=1,2, j=1,3) \\
& e_{i \mathrm{I}}^{n+1 / 2}=Z c_{\mathrm{r}} \Delta x^{0} d_{i \mathrm{I}}^{\mathrm{C}^{n+1 / 2}}, e_{i \mathrm{II}}^{n+1 / 2}=Z \frac{c_{\mathrm{r}} \Delta x^{0}}{2} d_{i \mathrm{II}}^{\mathrm{C}^{n+1 / 2}} \\
& e_{i \mathrm{II}}^{n+j / 4}=Z \frac{c_{\mathrm{r}} \Delta x^{0}}{4} \frac{d_{i \mathrm{II}}^{\mathrm{C}}}{\alpha},(i=1,2, j=1,3) \\
& d_{i K}^{\mathrm{C}} n+j / 4 \\
& =d_{i K}^{n+j / 4}+\frac{d_{12}^{n+j / 4}}{2} \quad(i=1,2, j=1,2,3, K=\mathrm{I}, \mathrm{II})
\end{aligned}
$$

where $d$ and $e$ are respectively the electric flux and the integration of the electric field given by (3), and $\alpha=1+$ $\left(c_{\mathrm{r}} \Delta x^{0}\right)^{2} / 16 \approx 1$. Variables are assigned as in Fig. 4. Based on the vectorial correction illustrated in Fig. 4(d), (6) gives a more accurate approximation of the constitutive equation than (5).

The constitutive relation between $d_{12}$ and $e_{12}$ are given by

$$
\begin{gathered}
e_{12}^{n+1 / 2}=Z \frac{c_{\mathrm{r}} \Delta x^{0}}{2} \frac{d_{12}^{n+1 / 2}}{2(1-\Delta l)}, e_{12}^{n+j / 4}=Z \frac{c_{\mathrm{r}} \Delta x^{0}}{4} \frac{d_{12}^{n+j / 4}}{2(\alpha-\Delta l)} \\
(j=1,3) .
\end{gathered}
$$

However, (6), (7), and (8) result in an asymmetric construction of the impedance matrix, which could cause a numerical instability [10]. To symmetrize the impedance matrix, (8) is replaced by

$$
\begin{gathered}
e_{12}^{n+1 / 2}=Z \frac{c_{\mathrm{r}} \Delta x^{0}}{2}\left(\beta d_{12}^{n+1 / 2}+d_{1 \mathrm{I}}^{n+1 / 2}+d_{2 \mathrm{I}}^{n+1 / 2}+\frac{d_{1 \mathrm{II}}^{n+1 / 2}}{2}+\frac{d_{2 \mathrm{II}}^{n+1 / 2}}{2}\right), \\
e_{12}^{n+j / 4}=Z \frac{c_{\mathrm{r}} \Delta x^{0}}{4}\left(\gamma d_{12}^{n+j / 4}+\frac{d_{1 \mathrm{II}}^{n+j / 4}}{2 \alpha}+\frac{d_{2 \mathrm{II}}^{n+j / 4}}{2 \alpha}\right) \quad(j=1,3)
\end{gathered}
$$

where $\beta=(5 / 2-3 \Delta l) /(2-2 \Delta l)$ and $\gamma=(3 / 2-\Delta l / \alpha) /(2 \alpha-2 \Delta l) \approx$ $(3 / 2-\Delta l) /(2-2 \Delta l)$. The correction with $(6)$ and $(9)$ is called the symmetric correction in this paper, whereas the correction with (6) and (8) is called the asymmetric correction. Relation (5) gives a diagonal impedance matrix without correction. An asymmetric correction was proposed in [8] for type-B grids (see Appendix A).

\section{B. Stability Analysis}

The grid in Fig. 1 has a period $\Delta x^{0}$ along the $x^{0}$-direction. Consequently the variables consisting of $f$ and $g$ given by (3) are periodically allocated along the $x^{0}$-direction on the spacetime grid. The variables are accordingly denoted $\boldsymbol{V}^{0}, \boldsymbol{V}^{1}, \ldots$, where $\boldsymbol{V}^{n+1}$ is assigned after $\boldsymbol{V}^{n}$ by the time-interval $\Delta x^{0}$. The variable vector $\boldsymbol{V}^{n}$ is divided into $\boldsymbol{v}^{n}$ and $\boldsymbol{u}^{n}$ where the components of $\boldsymbol{v}^{n}$ are linearly independent and the components of $\boldsymbol{u}^{n}$ are given by the linear combination of the components of $\boldsymbol{v}^{n}$. The numerical stability of the time-marching scheme is evaluated using the eigenvalues of $\partial \boldsymbol{v}^{n+1} / \partial \boldsymbol{v}^{n}$. A small spacetime grid having spatial domain size of $40 \times 40$ containing domain (II) with $18 \times 18$ spatial cells is used for the numerical eigenvalue analysis imposing spatially periodic boundary conditions. If $\Delta x^{0}$ is small, all the eigenvalues of $\partial \boldsymbol{v}^{n+1} / \partial \boldsymbol{v}^{n}$ given by the type-A grid are on the unit circle. If $\Delta x^{0}$ is large, some of the eigenvalues move outside the unit circle, causing numerical instability. Setting $\Delta l=5 / 6$, Fig. 5 contrasts the maximum $\Delta x^{0} / c$ that does not induce instability for the different grid types and corrections. Note that a type-B grid with correction yields eigenvalues for which the absolute values slightly exceed 1 by about $10^{-4}$, even with smaller $\Delta x^{0} / c$ than that shown in Fig. 5, that will cause late-time instability [9], [10]. Consequently, a type-B grid should not be used for lengthy computations with correction, even though a late-time instability was not found in [8]. In contrast, a type-A grid with symmetric or asymmetric correction increases the maximum time-step without inducing a late-time instability. As is shown in Appendix B, the scheme for a type-A grid with symmetric or asymmetric correction is equivalent to that for type-C, which is unaffected by the choice between (8) and (9). (a)
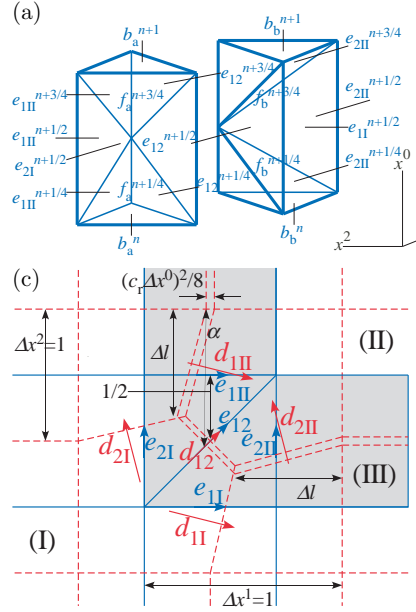

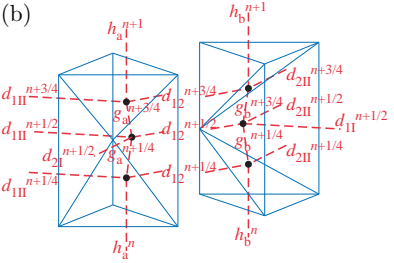

(d)

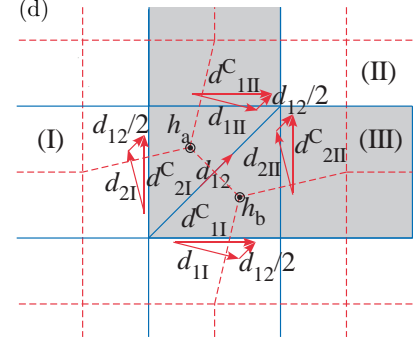

Fig. 4. Corner of domain (III) of a type-A grid: (a) variables on a primal grid, (b) variables on a dual grid, (c) directions of variables, and (d) vectorial correction.

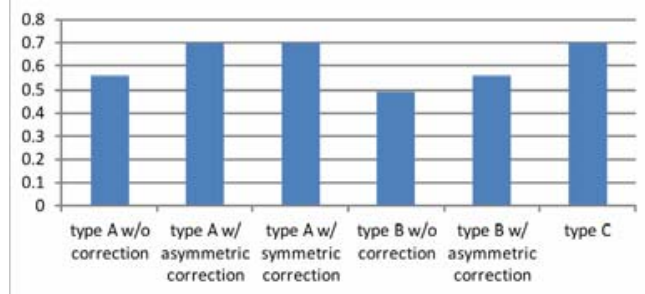

Fig. 5. Maximum $\Delta x^{0} / c$ for stable computation with respect to grid type and correction 

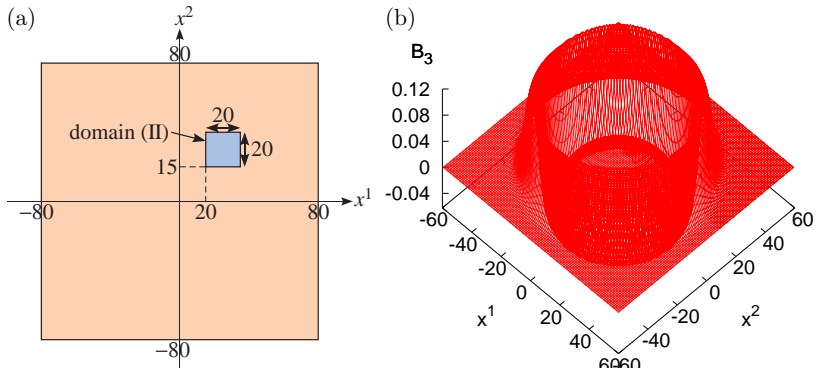

Fig. 6. Wave propagation computation; (a) computational domain and (b) distribution of $\mathscr{B}_{3}$ at $x^{0}=40$.
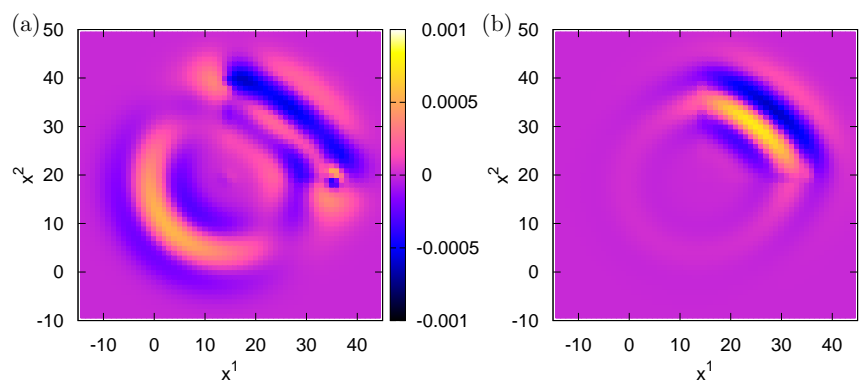

Fig. 7. Discrepancy in $\mathfrak{B}_{3}$ between the space-time FI method on a type-A grid and the FDTD method: (a) without and (b) with symmetric correction.

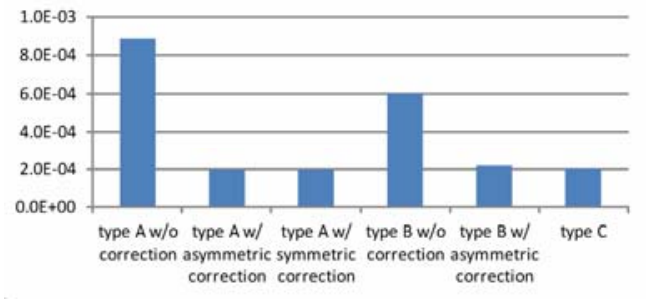

Fig. 8. Discrepancy of $\mathfrak{B}_{3}$ for the FDTD method in $x^{1} \leq 15$ or $x^{2} \leq 20$ of domain (I) with respect to grid type and correction.
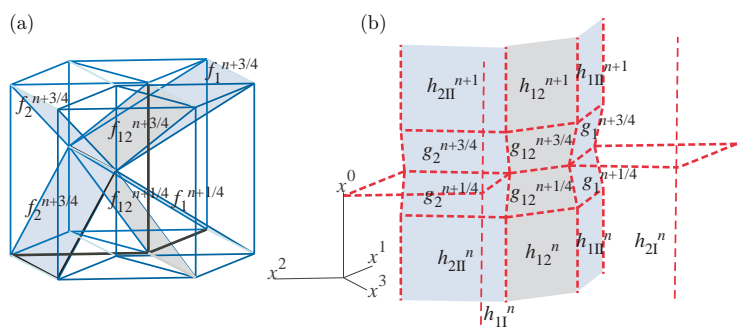

Fig. 9. 4D Space-time grid of type A: (a) primal grid, and (b) dual grid.

\section{Unphysical Wave Reflection}

Wave propagation is simulated to examine the three types of grid on the computational domain (Fig. 6(a)). For simplicity, the permittivity and permeability are set uniformly to unity by normalization. The normalized initial condition is given by $E_{1}$ $=E_{2}=0$ and $\mathfrak{B}_{3}=\exp \left\{-\left[\left(x^{1}\right)^{2}+\left(x^{2}\right)^{2}\right] / 25\right\}$. Spatially periodic boundary conditions are imposed. The time-step $\Delta x^{0}$ is set to 0.5 in domain (I) and $\Delta l$ is set to 5/6. Fig. 6(b) depicts the distribution of $\mathcal{B}_{3}$ at $x^{0}=40$.

Fig. 7 depicts the distributions of discrepancy $\Delta \mathfrak{B}_{3}$ between $\mathscr{B}_{3}$ at $x^{0}=40$ obtained by the FDTD method and the spacetime FI method with a type-A grid with and without symmetric correction. The FDTD method is executed with the same uniform spatial grid and time-step as in domain (I). The discrepancy seen in $x^{1} \geq 15$ and $x^{2} \geq 20$ is mainly caused by numerical dispersion whereas that in $x^{1} \leq 15$ or $x^{2} \leq 20$ is caused by an unphysical wave reflection at domain (III). Fig. 8 compares the latter discrepancy in domain (I). It shows that both the asymmetric and symmetric corrections effectively reduce the unphysical wave reflection equally to the type-C grid. The type-B grid also achieves accurate results under correction, but suffers a late-time instability before $10^{6} \Delta x^{0}$.

\section{4D-SPACE-TIME GRID WITH 3D SPACE}

The computational domain shown in Fig. 6(a) is extended to the $x^{3}$-direction for a 4D simulation. Fig. 9 illustrates a 4D space-time grid of type-A at the corner of domain (III). For the 4D computation, two types of constitutive relations given by (10) and (11) are examined as well:

$$
\begin{aligned}
& h_{i \mathrm{I}}^{n}=\frac{c_{\mathrm{r}} \Delta x^{0} \Delta l^{\prime}}{Z} b_{i \mathrm{I}}^{n}, h_{i \mathrm{II}}^{n}=\frac{c_{\mathrm{r}} \Delta x^{0} \Delta l}{2 Z} b_{i \mathrm{II}}^{n}, \\
& g_{i}^{n+j / 4}=\frac{c_{\mathrm{r}} \Delta x^{0} \Delta l}{4 Z} f_{i}^{n+j / 4},(i=1,2, j=1,3) \\
& h_{i \mathrm{I}}^{n}=\frac{\eta c_{\mathrm{r}} \Delta x^{0}}{Z}\left[b_{i \mathrm{I}}^{n}+(-1)^{i}(1-\Delta l) b_{12}^{n}\right], \\
& h_{i \mathrm{II}}^{n}=\frac{c_{\mathrm{r}} \Delta x^{0}}{2 Z}\left[\alpha b_{i \mathrm{II}}^{n}+(-1)^{i}(\alpha-\Delta l) b_{12}^{n}\right], \\
& g_{i}^{n+j / 4}=\frac{c_{\mathrm{r}} \Delta x^{0}}{4 Z}\left[\alpha^{\prime} f_{i}^{n+j / 4}+(-1)^{i}\left(\alpha^{\prime}-\Delta l\right) f_{12}^{n+j / 4}\right], \\
& (i=1,2, j=1,3)
\end{aligned}
$$

where $b$ and $h$ are the magnetic flux and the integration of magnetic field given by (3), respectively; $f$ and $g$ allocated as in Fig. 9 are also given by (3); $\Delta l^{\prime}=\Delta l-3\left(c_{\mathrm{r}} \Delta x^{0}\right)^{2} / 32 \approx \Delta l, \eta=$ $\Delta l^{\prime} / \Delta l \approx 1$ and $\alpha^{\prime}=(1+\alpha) / 2 \approx 1$. Based on a vector correction, (11) gives a more accurate approximation of the constitutive equation than (10) that gives a diagonal impedance matrix without correction.

The constitutive relations for $h_{12}$ and $g_{12}$ are given by

$$
\begin{aligned}
& h_{12}^{n}=\frac{c_{\mathrm{r}} \Delta x^{0}}{2 Z}(2 \alpha-2 \Delta l) b_{12}^{n}, \\
& g_{12}^{n+j / 4}=\frac{c_{\mathrm{r}} \Delta x^{0}}{4 Z}\left(2 \alpha^{\prime}-2 \Delta l\right) f_{12}^{n+j / 4} \quad(j=1,3) .
\end{aligned}
$$

To avoid asymmetric impedance matrix, (12) is replaced by

$$
\begin{gathered}
h_{12}^{n}=\frac{c_{\mathrm{r}} \Delta x^{0}}{2 Z}\left[\zeta b_{12}^{n}-2 \eta(1-\Delta l)\left(b_{1 \mathrm{I}}^{n}-b_{2 \mathrm{I}}^{n}\right)-(\alpha-\Delta l)\left(b_{1 \mathrm{II}}^{n}-b_{2 \mathrm{II}}^{n}\right)\right] \\
g_{12}^{n+j / 4}=\frac{c_{\mathrm{r}} \Delta x^{0}}{4 Z}\left(\alpha^{\prime}-\Delta l\right)\left(3 f_{12}^{n+j / 4}-f_{1 \mathrm{II}}^{n+j / 4}+f_{2 \mathrm{II}}^{n+j / 4}\right) \\
(j=1,3)
\end{gathered}
$$

where $\zeta=3(\alpha-\Delta l)+2 \eta(1-\Delta l) \approx 5(1-\Delta l)$. The correction with (6), (9), (11), and (13) is called the symmetric correction whereas the correction with (6), (8), (11), and (12) is called the asymmetric correction.

Wave propagation is simulated with $c=1, \Delta l=5 / 6$, and normalized initial conditions given by $E_{1}=E_{2}=E_{3}=0, \Re_{1}=$ $\exp \left\{-\left[\left(x^{2}\right)^{2}+\left(x^{3}\right)^{2}\right] / 25\right\}, \mathscr{B}_{2}=\exp \left\{-\left[\left(x^{3}\right)^{2}+\left(x^{1}\right)^{2}\right] / 25\right\}$, and $\mathfrak{B}_{3}=$ $\exp \left\{-\left[\left(x^{1}\right)^{2}+\left(x^{2}\right)^{2}\right] / 25\right\}$. For the different grid types and corrections, Fig. 10 contrasts the maximum $\Delta x^{0}$ that does not 
induce an apparent instability before $10^{4} \Delta x^{0}$. A type-A grid with symmetric and asymmetric corrections increases the maximum time-step. The type-A grid with the symmetric correction and the type- $\mathrm{C}$ grid do not develop numerical instability even after $10^{6} \Delta x^{0}$ if $\Delta x^{0} \leq 0.57$. However type-A and type-B grids with asymmetric correction suffer late-time instability, even with smaller $\Delta x^{0}$ than that shown in Fig. 10. Fig. 11 depicts the distribution for $|\mathfrak{B}|$ at $x^{0}=40$ given by a type-A grid with symmetric correction and the discrepancy $|\Delta \mathscr{B}|$ when compared with that from the FDTD method. Fig. 12 contrasts the discrepancy in domain (I) when $\Delta x^{0}=0.5$ over grid type and correction. Fig. 12 shows that both the asymmetric and symmetric corrections effectively reduce the unphysical wave reflection.

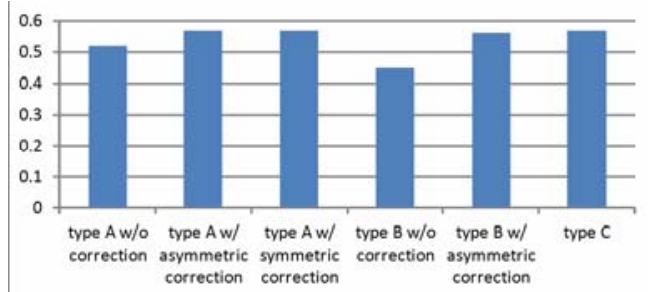

Fig. 10. Maximum $\Delta x^{0}$ for stable computation. (a)

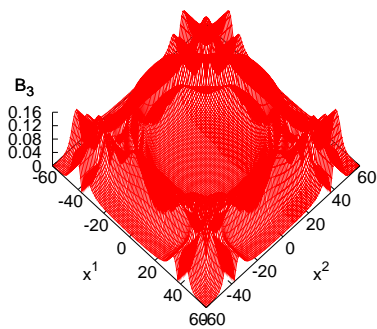

Fig. 11. Computation result on type A grid with symmetric correction; (a) distribution of $|\mathfrak{B}|$, and (b) Discrepancy $|\Delta \mathscr{B}|$ compared with FDTD method.

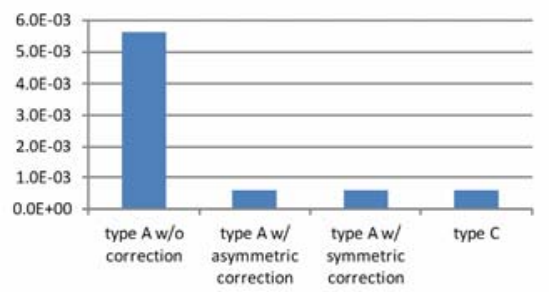

Fig. 12. Discrepancy of $\mathfrak{B}_{3}$ for the FDTD method in $x^{1} \leq 15$ or $x^{2} \leq 20$ of domain (I) with respect to grid type and correction.

\section{CONCLUSION}

A symmetric correction is proposed for the constitutive relation on a space-time grid that effectively reduces the unphysical wave reflection without numerical instability. Numerical examination shows that an asymmetric correction can induce late-time instability. In practice, the type-C grid provides a simple, stable, and accurate scheme for the spacetime FI method.

\section{APPENDIX}

\section{A. Correction for type $B$ grid}

The correction given by (9) and (10) in [8] is unfortunately not correct, where (9) and (10a) should have similar form to (5) and the first and second equations of (6) in this paper; $\varepsilon_{\mathrm{r}} \varepsilon_{0}$ in $(10 \mathrm{~b})$ in [8] should be replaced by $Z c_{\mathrm{r}} \Delta x^{0} / 4$.

\section{B. Equivalence to type $C$ grid}

Variables $d_{12}{ }^{n+j / 4}(j=1,2,3)$ are updated as

$$
\begin{aligned}
& d_{12}^{n+1 / 4}=d_{12}^{n-1 / 4}+h_{\mathrm{a}}^{n}-h_{\mathrm{b}}^{n}, \\
& d_{12}^{n+1 / 2}=d_{12}^{n+1 / 4}+g_{\mathrm{a}}^{n+1 / 4}-g_{\mathrm{b}}^{n+1 / 4}, \\
& d_{12}^{n+3 / 4}=d_{12}^{n+1 / 2}+g_{\mathrm{a}}^{n+3 / 4}-g_{\mathrm{b}}^{n+3 / 4}
\end{aligned}
$$

By setting, $b_{\mathrm{O}}{ }^{n}=b_{\mathrm{a}}{ }^{n}+b_{\mathrm{b}}{ }^{n}, \quad h_{\mathrm{O}}{ }^{n}=\left(h_{\mathrm{a}}{ }^{n}+h_{\mathrm{b}}{ }^{n}\right) / 2=$ $\left(c_{\mathrm{r}} \Delta x^{0} / 2 Z\right) b_{\mathrm{O}}{ }^{n}, f_{\mathrm{O}}{ }^{n+j / 4}=f_{\mathrm{a}}^{n+j / 4}+f_{\mathrm{b}}{ }^{n+j / 4}, g_{\mathrm{O}}{ }^{n+j / 4}=\left(g_{\mathrm{a}}{ }^{n+j / 4}+g_{\mathrm{b}}{ }^{n+j / 4}\right) /$ $2=\left(c_{\mathrm{r}} \Delta x^{0} / 4 Z\right) f_{\mathrm{O}}{ }^{n}(j=1,3), d^{\mathrm{C}}, f_{\mathrm{O}}$, and $b_{\mathrm{O}}$ are updated as

$$
\begin{aligned}
& d_{1 \mathrm{I}}^{\mathrm{C}^{n+1 / 2}}=d_{1 \mathrm{I}}^{\mathrm{C}^{n-1 / 2}}+\left(g_{\mathrm{O}}^{n+1 / 4}+h_{\mathrm{O}}^{n}+g_{\mathrm{O}}^{n+3 / 4}-h_{\mathrm{S}}^{n}\right), \\
& d_{1 \mathrm{II}}^{\mathrm{C} n+1 / 4}=d_{1 \mathrm{II}}^{\mathrm{C}^{n-1 / 4}}+\left(h_{\mathrm{N}}^{n}-h_{\mathrm{O}}^{n}\right), \\
& d_{1 \mathrm{II}}^{\mathrm{C}}{ }^{n+1 / 2}=d_{1 \mathrm{II}}^{\mathrm{C} n+1 / 4}+\left(g_{\mathrm{N}}^{n+1 / 4}-g_{\mathrm{O}}^{n+1 / 4}\right), \\
& d_{1 \mathrm{II}}^{\mathrm{C}}{ }^{n+3 / 4}=d_{1 \mathrm{II}}^{\mathrm{C}}{ }^{n+1 / 2}+\left(g_{\mathrm{N}}^{n+3 / 4}-g_{\mathrm{O}}^{n+3 / 4}\right) \\
& f_{\mathrm{O}}^{n+1 / 4}=b_{\mathrm{O}}^{n}-e_{2 \mathrm{II}}^{n+1 / 4}+e_{1 \mathrm{II}}^{n+1 / 4}, \\
& f_{\mathrm{O}}^{n+3 / 4}=f_{\mathrm{O}}^{n+1 / 4}-e_{2 \mathrm{II}}^{n+1 / 2}+e_{1 \mathrm{II}}^{n+1 / 2}+e_{2 \mathrm{I}}^{n+1 / 2}-e_{1 \mathrm{I}}^{n+1 / 2}, \\
& b_{\mathrm{O}}^{n+1}=f_{\mathrm{O}}^{n+3 / 4}-e_{2 \mathrm{II}}^{n+3 / 4}+e_{1 \mathrm{II}}^{n+3 / 4}
\end{aligned}
$$

where subscripts $\mathrm{N}$ and $\mathrm{S}$ indicate the positions shown in Fig. 2(b); $d^{\mathrm{C}}{ }_{2 K}{ }^{n+j / 4}$ ( $\left.K=\mathrm{I}, \mathrm{II}\right)$ are similarly updated using $h_{\mathrm{W}}, h_{\mathrm{E}}$, and $\mathrm{g}_{\mathrm{E}}$. The scheme above is equivalent to that for a type-C grid.

\section{REFERENCES}

[1] T. Weiland, "Time domain electromagnetic field computation with finite difference methods," Int. J. Numer. Model, vol. 9, pp. 295-319, 1996.

[2] I. E. Lager, E. Tonti, A.T. de Hoop, G. Mur, and M. Marrone, "Finite formulation and domain-integrated field relations in electromagnetics - a synthesis," IEEE Trans. Magn., vol. 39, pp. 1199-1202, May 2003.

[3] L. Codecasa and M. Politi, "Explicit, consistent, and conditionally stable extension of FD-TD to tetrahedral grids by FIT," IEEE Trans. Magn., vol. 44, pp. 1258-1261, June 2008.

[4] T. Matsuo, "Electromagnetic field computation using space-time grid and finite integration method," IEEE Trans. Magn., vol. 46, pp. 32413244, Aug. 2010.

[5] T. Matsuo, "Space-time finite integration method for electromagnetic field computation," IEEE Trans. Magn., vol. 47, pp. 1530-1533, Apr. 2011.

[6] T. Shimoi, T. Mifune, T. Matsuo, Y. Tanaka, "Finite integration method using local fine grid for explicit electromagnetic field computation," Digests 15th CEFC, TP2-4, p. 201, 2012.

[7] T. Matsuo, S. Shimizu, and, T. Mifune, "3D and 4D space-time grids for electromagnetic wave computation using finite integration method," APS-URSI Chicago, 362.3, 2012.

[8] T. Matsuo, and, T. Mifune, "3D and 4D space-time grids for electromagnetic field analysis using finite integration method," J. Jpn. Soc. Sim. Tech., vol.4, pp.176-180, 2012.

[9] S.D. Gedney and J.A. Roden, "Numerical stability of nonorthogonal FDTD methods," IEEE Trans. Antennas Propagat., vol. 48, pp. 231-239, Feb., 2000.

[10] R. Schuhmann and T. Weiland, "Stability of the FDTD algorithm on nonorthogonal grids related to the spatial interpolation scheme," IEEE Trans. Magn., vol. 34, pp. 2751-2754, May, 1998. 